\title{
D-brane instantons and matrix models
}

\author{
Iñaki García-Etxebarria \\ Department of Physics and Astronomy, University of Pennsylvania, \\ Philadelphia, PA 19104-6396, USA \\ E-mail: inaki@sas.upenn.edu
}

\begin{abstract}
We point out that in some situations it is possible to use matrix model techniques a la Dijkgraaf-Vafa to perturbatively compute D-brane instanton effects. This provides an explanation in terms of stringy instantons of the results in hep-th/0311181. We check this proposal in some simple scenarios. We point out some interesting consequences of this observation, such as the fact that it gives a perturbative way of computing stringy multi-instanton effects. It also provides a further interpretation of D-brane instantons as residual instantons of higgsed supergroups.
\end{abstract}




\section{Contents}

1. Introduction

2. Background material 3

2.1 The Dijkgraaf-Vafa correspondence

2.2 D-brane instantons 8

3. The glueball superpotential for low rank and D-brane instantons 10

4. A quiver example: $\mathbb{Z}_{n}$ orbifold of the conifold 13

5. Conclusions and further directions 15

\section{Introduction}

In a series of remarkable papers [1, 2, [3], Dijkgraaf and Vafa have argued that the exact low energy superpotential for a class of gauge theories can be computed by taking the planar limit of a matrix model. This matrix model is determined in a simple way by the deconstruction [4] of the original supersymmetric theory. It has a set of (bosonic) matrix valued fields in one to one correspondence with the superfields of the original theory, and a potential given by the superpotential of the original theory. For completeness, we include a short review of the Dijkgraaf-Vafa results in Section 2.1.

Remarkably, even if the effective superpotential is obtained from the planar limit of the matrix model, it is the low energy superpotential for finite $N$ in the original theory (with $N$ not necessarily large). A particularly interesting case is that in which $N$ is small. This case led originally to some puzzles, since for some particular cases the results obtained from the matrix model and the pure gauge theory seemed to disagree. The situation was clarified in [0], 6], where it was argued that the matrix model results might indeed differ from the field theory expectations for gauge factors of low rank, but the disagreement could be attributed to the choice of a UV definition of the gauge theory: the matrix model gives results for the definition of the gauge theory corresponding to the particular geometric embedding of the gauge theory in string theory chosen by Dijkgraaf and Vafa.

In [5, 6] the UV completion of the gauge theory was defined by its embedding in a (higgsed) supergroup of high enough rank. At the level of F-terms this definition of the gauge theory coincides with the usual gauge theory one, but it has the advantage that it is well defined in the UV. It is also very natural from the stringy point of view, where it can be understood as adding to the original DV embedding of the gauge theory an infinite set of brane-antibrane pairs with trivial K-theory charge. 
The purpose of this note is to point out, in the simplest possible set of examples, that it is also possible to understand this discrepancy between gauge theory and matrix models as coming from stringy D-brane instanton effects [7, 8, 9]. Our claim is that the matrix model computation includes the contribution of D-brane instantons to the low energy superpotential of the string theory realization of the gauge theory.

More precisely, we claim that it is possible to obtain the contributions of D-brane instantons to the effective superpotential by studying the planar limit of the associated matrix model. In the conclusions we include some preliminary remarks about the interpretation of the non-planar contributions, and we also make some comments on how could it be possible to generalize our results to some geometries beyond the Dijkgraaf-Vafa setup.

An interesting consequence of this result is that it gives a relatively easy and systematic way of computing perturbatively stringy multi-instanton effects, at least when a matrix model description of the system is available. These multi-instanton effects are typically rather involved to study using stringy instanton calculus, so the perturbative approach based on the matrix model will be a welcome tool.

We can find some other interesting implications of the result. There are by now different ways of computing exotic D-brane instanton effects by modifying the system such that it admits a gauge theory interpretation while still allowing to extract the relevant information. Let us mention as examples 10, 11, 12, in which Seiberg duality (as in the duality cascade) is used to turn D-brane instantons into conventional gauge non-perturbative effects. Another example is [13, where Seiberg duality is combined with higgsing.

From this perspective, the matrix model viewpoint offers yet another embedding of Dbrane instanton effects into gauge theory. It is given in terms of the supergroup construction of [5], which essentially consists of adding infinite brane-antibrane pairs to the system. In this setup stringy D-brane instanton effects are identified with residual instanton effects of the higgsed supergroup.

Some of the constructions above also admit a nice interpretation in terms of matrix models. For example, as explained in [3], Seiberg duality in the case of ADE quiver theories can be understood as Weyl reflections of the quiver diagram, and the matrix model results are essentially insensitive to this Weyl reflection. Similarly, as described in [4], the F-terms computed by the matrix model are insensitive to where we are in the duality cascade, so we can do our calculations either "up the cascade", where everything can be formulated in terms of gauge theory, or at the bottom of the cascade, where we have exotic D-brane instanton effects with no gauge theory interpretation. Let us mention that 泪 also provides a nice intermediate viewpoint between the brane-antibrane configuration and the matrix model in the form of the supermatrix model description, which turns out to be rather useful for understanding the physics of these duality cascades in the context of matrix models.

We would also like to point out that very similar considerations to some of the ones we discuss here have already appeared in [14]. In that paper, the geometric transition is used to compute non-perturbative effects due to stringy D-brane instantons. The matrix model 
and the geometric transition approaches are very closely related, and they can be shown to give the same result in the planar limit, which determines the superpotential. This is, nevertheless, a large $N$ effect ${ }^{1}$, and we expect that we have to use the matrix model for computing higher derivative interactions in the effective superpotential. If we restrict ourselves to the superpotential, and thus the planar limit, the matrix model provides a different but equivalent viewpoint of the discussion in [14].

We feel that the previous reasons make it worth pointing out the explicit connection between D-brane instantons and matrix models. It is, in any case, a subtle and interesting connection between two rich fields of study.

Let us mention that in this note we will be checking the proposed connection only in the simplest possible scenario: we will be computing one-instanton superpotential contributions for quiver gauge theories admitting a matrix model description a la Dijkgraaf and Vafa. This leaves many issues to be studied, and we hope to come back to them in future work.

The remainder of this note is organized as follows. We start in Section 2 by summarizing the main points of the Dijkgraaf-Vafa correspondence and D-brane instanton calculus. In Section 5 we see how the D-brane instanton and matrix model results agree for the simplest one-instanton sector in conifold geometries. In Section 4 we study a more interesting and involved example, the abelian orbifold of the conifold. Section 5 contains our conclusions and some discussion of interesting open problems.

\section{Background material}

\subsection{The Dijkgraaf-Vafa correspondence}

Let us start by reviewing the main results of Dijkgraaf and Vafa [1, 2, 股. Since we will be dealing with stringy instantons we concentrate on the stringy derivation and interpretation of the results, although the same results can also be derived in pure field theory [15, 16, 17].

\section{Gauge theory}

The configurations we will be concerned with are stringy embeddings of $\mathcal{N}=1$ gauge theories with a mass gap, the lightest states being glueball superfields ${ }^{2}$. The canonical example is a $\mathcal{N}=1$ theory obtained from $\mathcal{N}=2 U(N)$ SYM with no flavors and a potential for the adjoint superfield:

$$
\int d^{2} \theta \operatorname{Tr} W(\Phi)
$$

This superpotential breaks down the supersymmetry to $\mathcal{N}=1$. We take $W(z)$ to be a polynomial function of degree $n+1$. We will denote the dynamical scale of this theory by $\Lambda$.

\footnotetext{
${ }^{1}$ We hope that the terminology we use does not lead to confusion. The $N$ here refers to the rank of the matrices in the matrix model, and has nothing to do with the ranks of the physical gauge group. We review this point in Section 2.1.

${ }^{2}$ The existence of the mass gap, and glueballs being the correct low energy degrees of freedom, are statements which are very hard to show rigorously. Here we just assume them to hold.
} 
The classical vacua of these theory are obtained by extremizing the superpotential $W$. This superpotential has $n$ extrema $a_{i}$, let us indicate this by writing the derivative of $W$ as $W^{\prime}(z)=g \prod_{i=1}^{n}\left(z-a_{i}\right)$. A classical solution of $\operatorname{Tr} W^{\prime}(\Phi)=0$ is obtained by considering a partition of the $N$ eigenvalues of $\Phi$ into the $n$ extrema:

$$
\Phi=\left(\begin{array}{llllll}
a_{1} & & & & \\
& a_{1} & & & & \\
& & a_{2} & & & \\
& & a_{4} & & \\
& & & \ddots & \\
& & & & & a_{n}
\end{array}\right)
$$

Around any of these vacua $\Phi$ is massive so it can be integrated out of the low energy theory, which is then given by $\mathcal{N}=1 \mathrm{SYM}$ with the Higgsed gauge group $\prod_{i=1}^{n} U\left(N_{i}\right)$. The $N_{i}$ here count how many eigenvalues of $\Phi$ are equal to $a_{i}$. It is conventional and convenient to denote this Higgsing process $U(N) \rightarrow \prod_{i=1}^{n} U\left(N_{i}\right)$.

This theory confines, and it is believed that the low energy degrees of freedom of this theory after confinement, similarly to the case of pure SYM, are given by the glueball superfields $S_{i}$, one for each of the gauge factors:

$$
S_{i}=-\frac{1}{32 \pi^{2}} \operatorname{Tr}_{S U\left(N_{i}\right)} W_{\alpha} W^{\alpha}
$$

with $W_{\alpha}$ the spinorial chiral superfield appearing in the gauge kinetic action, having lowest component the gluino $\lambda_{\alpha}$. As explained by Veneziano and Yankielowicz [18], for pure SYM the low energy dynamics can be obtained from an effective superpotential involving the glueball superfield:

$$
W_{e f f}(S)=S \log \left(\Lambda^{3 N} / S^{N}\right)+N S
$$

where $\Lambda$ denotes the dynamical scale of the $S U(N)$ theory.

In the case of the theory we are dealing with, the effective superpotential will be of the form [1]:

$$
W_{e f f}(S)=\sum_{i=1}^{n}\left[S_{i} \log \left(\frac{\Lambda_{i}^{3 N_{i}}}{S_{i}^{N_{i}}}\right)+N_{i} S_{i}+N_{i} \frac{\partial \mathcal{F}_{0}(S)}{\partial S_{i}}\right]
$$

where $\mathcal{F}_{0}$ is a perturbative series on the glueballs $S_{i}$, and $\Lambda_{i}$ is the dynamical scale of the factor $S U\left(N_{i}\right)$ after integrating out the massive matter. The usual matching relations lets us express $\Lambda_{i}$ in terms of the dynamical scale $\Lambda$ of the original theory, the derivative of the superpotential $W^{\prime}(z)$, and the residual ranks $N_{i}$ :

$$
\Lambda_{i}^{3 N_{i}}=\Lambda^{2 N} g^{N_{i}} \prod_{j \neq i}\left(a_{j}-a_{i}\right)^{N_{i}-2 N_{j}}
$$

Similar matching relations for the related $S O(N) \rightarrow S O\left(N_{0}\right) \times \prod_{i} U\left(N_{i}\right)$ and $S p(N) \rightarrow$ $S p\left(N_{0}\right) \times \prod_{i} U\left(N_{i}\right)$ cases can be found in [6].

What Dijkgraaf and Vafa showed is that $\mathcal{F}_{0}$ can be computed perturbatively from a related matrix model. We describe the main points of their argument below, but let us first describe how we will embed these gauge theories into string theory. 


\section{Geometry}

A type IIB configuration realizing the gauge theory we are studying was described in [19]. It is given by Minkowski spacetime times a local Calabi-Yau defined by the resolution of:

$$
x^{2}+y^{2}+w^{2}+\left(W^{\prime}(z)\right)^{2}=0
$$

with $W(z)$ the same function as in (2.1), and $W^{\prime}(z)$ its first derivative with respect to $z$. The extrema of $W(z)$ are located at $z=a_{i}$, so we can rewrite (2.7) more clearly as:

$$
x^{2}+y^{2}+w^{2}+g^{2} \prod_{i=1}^{n}\left(z-a_{i}\right)^{2}=0 .
$$

It is easy to see from here that when $z$ is close to $a_{i}$ (we take the $a_{i}$ to be well separated for simplicity), the equation (2.8) reduces to the one for the conifold. We take the conifold to be resolved ${ }^{3}$, so the geometry is no longer singular, but instead has an isolated 2cycle at each of the degenerations $a_{i}$. The size of the resolved 2-cycles is determined by the complexified gauge coupling constant for the theory we are engineering, more on this below.

Let us consider this background in the presence of $N$ D5 branes, which we take to wrap Minkowski and two internal directions. The low energy dynamics of such a system is $U(N)$ with the superpotential (2.1) for the adjoints. Classical vacua of the system correspond to distributing the $N$ D-branes in different ways in the $n$ different 2-spheres of the resolved conifolds. When the $N$ branes get separated into the different cycles, some open strings get massive, and the gauge group on the branes factorizes. Furthermore the adjoint chiral multiplet living on the brane, and which parametrizes motions of the brane, gets a positive mass since the resolution two-sphere is a local volume minimum in its homology class.

Let us briefly mention that all the resolved two spheres belong to the same homology class. One simple way to see this in physics terms is that we can tunnel from $U\left(N_{1}\right) \times U\left(N_{2}\right)$ to $U\left(N_{1}-1\right) \times U\left(N_{2}+1\right)$ in the gauge theory just by smoothly taking one eigenvalue of $\Phi$ from $a_{1}$ to $a_{2}$. For such a tunneling to be possible in string theory the total homology class should stay the same, the $a_{1} \rightarrow a_{2}$ interpolation describes the 3-chain that makes the two cycles homologous. Such a tunneling is described by instantons of the matrix model [1, 21], which are related to domain walls in the effective theory interpolating between the two vacua, and coming from branes wrapping the 3-chain.

The bare holomorphic coupling constant $\tau$ of the Yang-Mills theory living on the brane is given by the complexified volume of the two cycle $S$ wrapped by the brane:

$$
\tau=\frac{\theta}{2 \pi}-\frac{4 \pi i}{g_{Y M}^{2}}=\frac{1}{2 \pi i g_{s}} \int_{S}(J+i B) .
$$

We will also be interested in engineering $S p(N)$ theories with a superpotential for the adjoint. They can be obtained by adding a suitable orientifold to the geometry above. The breaking pattern in this case is $S p(N) \rightarrow S p\left(N_{1}\right) \times U\left(N_{2}\right) \times \ldots \times U\left(N_{n}\right)$, but otherwise everything is very similar to the $U(N)$ case $[6]$.

\footnotetext{
${ }^{3}$ Resolution means, roughly, that we substitute the singularity of the conifold by a finite size $\mathbf{S}^{2}$ in such a way that the resulting space is smooth. See for example [20] for a more detailed description.
} 


\section{The low energy degrees of freedom of the string theory}

We see that up to now the tree level dynamics of the stringy system and the gauge theory are very similar, and can be matched quite easily. Nevertheless, we have not yet described what plays the role of the all-important glueball superfield in the string side. This is a subtle problem, and central to our discussion.

It turns out that in order to account for all the low energy dynamics of string theory one needs to include more "glueball" superfields in the string theory side than those that would be necessary just from the gauge theory point of view. In fact, one way of reading the results of this note is that these extra degrees of freedom in the string side are those necessary in order to account for the stringy instanton effects, which are not present in gauge theory.

These extra glueballs must be included for the cases in which there is a single brane wrapping a $\mathbf{S}^{2}$, giving rise to a $U(1)$ gauge group, and in the case on which the $\mathbf{S}^{2}$ is wrapped by no branes, but there is an orientifold such that the gauge group on the cycle, had there been $N$ branes wrapped on it, would have been $S p(N)$. We will refer to this last case as the $S p(0)$ case. In gauge theory, none of this two gauge groups would give rise to a glueball and a Veneziano-Yankielowicz superpotential describing its dynamics. These are also the only cases on which one has to include these extra degrees of freedom in order to match with the superpotential due to one stringy instanton effects. Other candidates one could think of such as $S O(0), S O(2)$ or $U(0)$ do not require extra glueballs for describing the stringy dynamics. The justification of this result from our point of view is that these are the D-brane instantons that contribute to the superpotential (see Section 2.2 below), so we need to make the corresponding glueballs dynamic in order to reproduce the known stringy instanton effects.

Note that the prescription given in [6] for when to include the extra stringy glueballs, which was obtained via the geometric transition, coincides with our prescription that the matrix model should compute D-brane instanton effects, which is a successful check of our proposal.

We should point out that the previous discussion is the correct one when we are restricting ourselves to superpotential contributions in the single instanton case (as we are doing in this note), but we expect that it might be necessary to consider dynamical at least the $U(0)$ glueballs in the description of more complicated processes. The reason is the following. Consider instantons in a $U(0)$ gauge group (these are commonly called $U(1)$ instantons in the D-brane instanton literature). They have four neutral zero modes, and thus they do not contribute to the superpotential by themselves, in agreement with the fact that we are considering the corresponding glueball non-dynamical. Nevertheless, as discussed in [11], multi-instanton processes involving a $S p(0)$ and a $U(0)$ instanton can contribute to the superpotential. We cannot reproduce these effects in the matrix model side if we just set the $U(0)$ glueball to zero from the beginning. One way out is to include the $U(0)$ glueball in the game, and just set to zero the Veneziano-Yankielowicz part of the superpotential. Namely, we take the following formula to hold for any $N \geq 0$ :

$$
W_{U(N)}=S \log \left(\Lambda_{0}^{3 N} / S^{N}\right)+N S-2 \pi i \tau S
$$


Here $\Lambda_{0}$ is the cutoff for the theory, which combines with the bare holomorphic coupling $\tau=\frac{\theta}{2 \pi}-\frac{4 \pi i}{g_{Y M}^{2}}$ in order to give the usual holomorphic scale $\Lambda$ of the gauge theory:

$$
\Lambda^{3}=\Lambda_{0}^{3} e^{-2 \pi i \tau / N}
$$

reproducing in this way the Veneziano-Yankielowicz superpotential (2.4).

Note that the first two terms in (2.10) vanish when $N=0$. For the one-instanton superpotential, such a prescription dynamically sets $S=0$ due to the effect of an extra light hypermultiplet appearing close to $S=0$ [6], but for multi-instanton processes there could be nontrivial contributions.

This prescription for the $U(0)$ glueball has a chance of matching the multi-instanton results, and also fits well with the heuristic idea that dynamical glueballs are associated with instanton effects in the corresponding node. We hope to come back to this interesting question in the future. In any case, we will not go beyond the one-instanton sector in this note, so this subtlety will not be important and we refrain from further discussion of this point.

\section{Matrix models as the theory on the branes}

Let us now come back to the connection between the string theory and the matrix model, following [1, 2, 3]. Since we are interested in computing F-terms, we can go to the topological string. As described above, we have $N$ D5 branes in a geometry defined by the function $W(z)$. The topological theory living on the worldvolume of the D5 branes is the reduction of three complex dimensional holomorphic Chern-Simons theory [22] to the two dimensional worldvolume of the brane. Partially solving the equations of motion for this theory tells us that the F-term physics is captured by the partition function of the holomorphic matrix model with action:

$$
\mathcal{Z}=\frac{1}{\operatorname{Vol}\left(U\left(M_{1}\right) \times \cdots \times U\left(M_{n}\right)\right)} \exp \left(-\frac{1}{g_{s}} \int d \Phi \operatorname{Tr} W(\Phi)\right)
$$

This formula requires some explanation. Here $\Phi$ is a $M \times M$ matrix ( $M$ is not related to the number of branes $N$, its relevance to the physical theory will be explained shortly), and $g_{s}$ is a overall scale unrelated to the coupling constant of the physical theory. As above, the classical vacua of this theory will be determined by extremizing $W(\Phi)$, and distributing the $M$ vacua into the $n$ extrema. We take $M=M_{1}+M_{2}+\ldots+M_{n}$. The prefactor takes into account the volume of the unbroken gauge group in the chosen vacuum.

For the particular case of the superpotential, which will be the main focus of this note, it can be further argued [23] that only the planar diagrams contribute ${ }^{4}$. This means that the required information can be obtained in the large $M$ limit of the matrix model. As usual when taking large $M$ limits, we will keep the 't Hooft coupling $S_{i}=g_{s} M_{i}$ fixed. This has two desirable consequences. First it tells us that we can solve the matrix model in

\footnotetext{
${ }^{4}$ The argument boils down to the fact that the planar diagrams are the ones without momentum insertions. It also implies that the higher F-terms, which do involve momentum insertions, are computed by the non-planar part of the matrix model. We will come back to this observation in the conclusions.
} 
the saddle point approximation, which is easier; and second, one can apply the arguments of [24, 25] to argue that the result can be computed via a geometric transition to a flux background.

Let us now describe how the prescription goes for connecting the matrix model amplitudes to F-terms in the physical string. The 't Hooft parameters $S_{i}$ of the matrix model get identified with the glueballs $S_{i}$ of the physical string. In this way, the perturbative expansion of the matrix model is an expansion in powers of the glueballs. The conjecture is that the low energy superpotential of the string theory is given by:

$$
W_{e f f}(S)=\sum_{i=1}^{n}\left[N_{i} \frac{\partial \mathcal{Z}_{0}(S)}{\partial S_{i}}-2 \pi i \tau_{i} S_{i}\right]
$$

where $N_{i}$ denotes the rank of the gauge factor $U\left(N_{i}\right)$ and $\tau_{i}$ is the bare holomorphic gauge coupling on the branes wrapping the cycle which is determined by the volume of the wrapped two cycle as in eq. $(2.9)^{5}$. $\mathcal{Z}_{0}$ denotes the planar part of the matrix model partition function (2.12).

Expressions (2.13) and (2.5) match nicely once we take into account the following. As explained in [26] from the mirror Chern-Simons perspective, the answer for the free planar part of the matrix model, once we take the volume of the residual gauge group into account, gives the Veneziano Yankielowicz superpotential. We can then expand the planar partition function into a free part and a correction:

$$
\mathcal{Z}_{0}=\left\{\frac{1}{\operatorname{Vol}\left(U\left(M_{1}\right) \times \cdots \times U\left(M_{n}\right)\right)} \exp \left(-\frac{1}{g_{s}} \int d \Phi \operatorname{Tr} \frac{\Phi^{2}}{2}\right)\right\}+\mathcal{F}_{0}
$$

The first term gives rise to the Veneziano-Yankielowicz term in the superpotential (notice also that it is the partition function for the conifold), and $\mathcal{F}_{0}$ is a polynomial in $S_{i}$ :

$$
\mathcal{F}_{0}=\sum_{i_{1} \ldots i_{n}} c_{i_{1} \ldots i_{n}} S_{1}^{i_{1}} \cdots S_{n}^{i_{n}}
$$

This series expansion in $S_{i}$ can be understood as a systematic fractional instanton expansion, where the power of $S_{i}$ determines the number of fractional instantons in the factor $U\left(N_{i}\right)$ we are taking into account. In this note we will be considering just the one instanton sector, which is determined by the universal term coming from the free (conifold) theory. We leave the study of multi-instanton effects due to the $\mathcal{F}_{0}$ expansion for future work.

\subsection{D-brane instantons}

Let us now proceed to review the aspects of D-brane instanton calculus most relevant to our discussion. As reviewed in the previous section, the Calabi-Yau has cycles over which we can wrap space-filling D-branes in order to engineer $4 \mathrm{~d}$ supersymmetric gauge theories.

\footnotetext{
${ }^{5}$ When computing instanton effects, sometimes there will be no spacetime filling D-brane wrapping the cycle, and thus the definition of $\tau$ as a gauge coupling constant does not make sense. The definition in terms of the (complexified) volume of the resolved cycle still makes sense, and it is the one we will always be implicitly using.
} 
Depending on the particular vacuum we choose there might also be cycles not wrapped by any spacetime filling D-brane. Over these cycles we might wrap D-brane instantons, which in this context are branes wrapping these internal cycles and localized at a point in spacetime. They give nonperturbative (in $g_{s}$ ) contributions to the $4 \mathrm{~d}$ effective action living on the spacetime wrapping branes.

The basic condition for a D-brane instanton to contribute to the superpotential in this setting is that it has exactly two unlifted fermionic zero modes, which will play the role of the $\theta$ variables in the integration of the superpotential over superspace. The kind of backgrounds where are considering preserve, in the absence of branes and orientifolds, 8 supercharges. A D-brane instanton is a $1 / 2$ BPS object, and thus has 4 fermionic zero modes $\theta_{\alpha}, \bar{\theta}_{\dot{\alpha}}$ corresponding to the 4 spontaneously broken supersymmetries. If the D-brane instanton is going to contribute to the superpotential, we should include some mechanism that lifts the two $\bar{\theta}_{\dot{\alpha}}$ Goldstinos.

The simplest configurations in which this happens, and which have no simple gauge theory interpretation, are instantons of the $U(1)$ and $S p(0)$ types. We describe them in the following.

An instanton of $S p(0)$ type occupies a cycle in which there are no wrapped space-filling branes, and which is mapped to itself by the orientifold action. Furthermore, the sign of the orientifold projection is such that the gauge group on the instanton is $O(1)$. Such a projection does indeed remove the $\bar{\theta}_{\dot{\alpha}}$ Goldstinos, as we want ${ }^{6}$.

In order to make the connection with the matrix model more transparent, here we have chosen to refer to this kind of instanton as " $S p(0)$ ", instead of using the more common denomination " $O(1)$ ". The justification for this denomination from the instanton point of view is the following. Consider the orientifold action on $N$ space-filling D-branes wrapping the same cycle as the instanton. Since the orientifold projection acts oppositely on space-filling and instanton branes, these branes would have gauge group $S p(N)$. The Dbrane instanton in this context can be interpreted as the gauge instanton for $S p(N)$, with the couplings for the string modes between the different branes implementing the ADHM construction [28, 29, 30, 31, 32]. The notation $S p(0)$ is thus intended to suggest that the D-brane instanton can be thought of as a gauge instanton of a (admittedly rather empty) $S p(0)$ gauge group.

Let us now describe instantons of the $U(1)$ type. These instantons do not exist in pure field theory either, but they do exist in string theory when there is a single D-brane wrapping the same cycle as the instanton [14, 11, 33.

In order to explain why this happens, let us consider first $N$ spacetime filling branes wrapping the cycle, giving a $U(N)$ gauge theory. Intuitively, since it is on top of the Dbranes, the D-brane instanton "feels" just $\mathcal{N}=1$, and thus it has the two desired Goldstinos $\theta_{\alpha}$ only. More precisely, the couplings between the zero modes of the instanton and the fields living in the branes implement the ADHM constraints, and the ADHM couplings lift the $\bar{\theta}_{\dot{\alpha}}$ modes.

\footnotetext{
${ }^{6}$ See for example [27] for a nice discussion of the details of the possible orientifold actions and their effect on the instanton zero modes
} 
One interesting observation one could make is that the way in which the couplings between the zero modes implement the ADHM constraints and lift the two extra goldstinos does not require that $N$ is bigger than 1 for $U(N)$, it works for $U(1)$ gauge theories too as studied in detail in [33]. $U(1)$ theories do not have instanton effects in gauge theory, and the fact that D-brane instantons contribute in this case is very much related to the fact that we are completing this $U(1)$ theory in the UV by embedding into string theory. To restate one of the main points of [6] and also of this work: such a completion is not as innocuous as it looks, since it also changes the low energy superpotential due to these exotic $U(1)$ instantons.

Before going on to check the agreement between the matrix model and the D-brane instanton calculus in these cases, let us make a couple of important remarks. Although we restrict here to the case in which the instanton has exactly two zero modes, the general case in which the instanton has more zero modes is also interesting. What happens here is that the instanton does not contribute to the superpotential, but can generate couplings involving more than two fermions, called higher F-terms [34, 35]. One important example is the gauge instanton for SQCD with $N_{f}=N_{c}$. These instantons do not contribute to the superpotentials, but induce operators with insertions of more than two fermions (or equivalently, derivatives). They are of the form:

$$
\int d^{4} x d^{2} \theta w_{\bar{i}_{1} \bar{j}_{1} \ldots \bar{i}_{n} \bar{j}_{n}}(\Phi, \bar{\Phi})\left(\overline{D \Phi}^{\bar{i}_{1}} \cdot \overline{D \Phi}^{\bar{j}_{1}}\right) \cdots\left(\overline{D \Phi}^{\bar{i}_{n}} \cdot \overline{D \Phi}^{\bar{j}_{n}}\right)
$$

where $\Phi$ represents the moduli of the system, and $\omega$ is a section of $\bar{\Omega}_{\mathcal{M}}^{p} \otimes \bar{\Omega}_{\mathcal{M}}^{p}$, with $\mathcal{M}$ the moduli space ${ }^{7}$. We include some preliminary remarks on the relation between matrix models and higher F-terms in the conclusions.

Another interesting possibility for lifting extra zero modes which we have not discussed here is by using fluxes, as studied in [36, 37, 38, 39, 40]. Modifying the matrix model side of the story in order to incorporate the effect of these fluxes is a worthwhile and interesting problem which we will not attempt to solve here.

\section{The glueball superpotential for low rank and D-brane instantons}

In this section we will study the two basic cases in which the matrix model result does not match the gauge theory result, namely, as reviewed in Section 2.1, configurations with gauge group $U(1)$ and $S p(0)$. We will see that there is a nice agreement between the result of the D-brane instanton calculation and the result obtained from the matrix model prescription. This gives evidence for the advertised result that matrix models can be used to compute D-brane instanton effects.

The geometry we will be focusing on will be the resolved conifold, so let us take a quadratic superpotential for the gauge theory:

$$
\int d^{2} \theta \operatorname{Tr} W(\Phi)=\int d^{2} \theta \operatorname{Tr} \frac{\Phi^{2}}{2}
$$

\footnotetext{
${ }^{7}$ In other words, it is a form antisymmetric in the $\bar{i}$ indices, antisymmetric in the $\bar{j}$ indices, and these indices live in the cotangent bundle over the moduli space.
} 
where the factor of $1 / 2$ is for later convenience. Plugging this $W(x)$ into equation (2.7) we obtain the conifold equation $x^{2}+y^{2}+z^{2}+w^{2}=0$. As explained above, we will be considering the resolved conifold, with a resolution parameter determined by the theory we are engineering.

\section{The matrix model computation}

Let us start by the matrix model side of the story, which is the simplest. We have to compute the planar limit of the matrix model free energy in this background. The conifold is described by the free holomorphic one-matrix model, so we just obtain the VenezianoYankielowicz result. Note that we must set $N=1$ in eq. (2.5) in order to capture $S p(0)$ and $U(1)$ D-brane instanton effects, as discussed in Section 2.1. We get:

$$
W_{e f f}(S, \tau)=S \log \left(\frac{\Lambda_{0}^{3}}{S}\right)+S-2 \pi i \tau S
$$

with $\Lambda_{0}$ the cutoff scale and $\tau$ the bare coupling of the configuration, defined in eq. (2.9):

$$
2 \pi i \tau=\frac{1}{g_{s}} \int_{\mathbf{S}^{2}} J+i B
$$

We can now proceed to integrate $S$ out. Its equation of motion gives:

$$
S=e^{-2 \pi i \tau} \Lambda_{0}^{3}
$$

which upon substitution into (3.2) gives:

$$
W=e^{-2 \pi i \tau} \Lambda_{0}^{3}=\Lambda^{3}
$$

where we have introduced the cutoff independent scale $\Lambda$.

\section{The D-brane instanton computation}

Let us now describe in some detail how the stringy instanton result reproduces this contribution. In order to avoid the complications of having to impose the (super)ADHM constraints, we will focus on the $S p(0)$ case, so the $\bar{\theta}$ modes are simply projected out by the orientifold. A good place to see how the saturation of the $\bar{\theta}$ modes goes for $U(1)$ instantons is for example 33].

We will start by considering the case with $W=0$. In this case we will have 8 background supercharges close to the orientifold, and 16 in the bulk. The $S p(0)$ instanton is a 1/2 BPS object wrapping a 2-cycle mapped to itself under the orientifold action, so it

will have 4 Goldstinos in its worldvolume. Let us call the surviving Goldstinos $\theta_{\alpha}^{A}$, where $A$ is an index taking values in the fundamental representation of the $S U(2)$ R-symmetry group preserved by the orientifold. Such an instanton has four neutral zero modes, and thus cannot contribute to the superpotential.

We now switch on the quadratic superpotential $W(z)=\frac{1}{2} m z^{2}$. This reduces the supersymmetry of the background by half, so now we have 8 supercharges on the bulk and 4 close to the orientifold. In such a configuration it is reasonable to guess that two of the 
four zero Goldstinos of the instanton get lifted, and the instanton can now contribute to the superpotential. This is indeed the case, let us now explain how this deformation acts from the point of view of the effective action on the instanton zero modes.

Let us start by studying the case were we have background spacetime filling branes, so we are dealing with ordinary gauge theory instantons. As reviewed in Section 2.1, in the gauge theory the deformation of the background appears as a breaking of the supersymmetry from $\mathcal{N}=2$ to $\mathcal{N}=1$ by the addition of the superpotential for the adjoint chiral multiplet:

$$
W(\Phi)=\frac{1}{2} m \operatorname{Tr} \Phi
$$

One consequence of such a coupling is that some of the zero modes associated to the field $\Phi$ get lifted, let us call such modes $\lambda_{\alpha}$ and $\bar{\lambda}_{\dot{\alpha}}$. We have chosen this notation in order to remind the reader that the adjoint superfield comes from the vector multiplet of $\mathcal{N}=2$, so the highest component fermion of the multiplet can be thought of as a gaugino from the point of view of the broken $\mathcal{N}=1$ algebra. As reviewed in Section VI.4 of [41], this can be nicely encoded in the effective action for the instanton zero modes as the addition of the following term:

$$
\delta S_{\text {inst }}=-\frac{m \pi^{2}}{g_{Y M}} \lambda^{\alpha} \lambda_{\alpha}
$$

Such a term can be used to saturate the $d^{2} \lambda$ term in the instanton measure, allowing the instanton to contribute to the superpotential.

We now claim that a term similar to (3.7) also exists for our $S p(0)$ instanton. We cannot compute the existence of such a coupling via CFT methods in string theory, but given the fact that string theory gives rise to the whole ADHM construction in such a natural way 42, 43], with a natural identification of the instanton-instanton strings as gauge theory zero modes, assuming that an analog of (3.7) holds seems to be reasonable.

From the point of view of the instanton brane $\lambda_{\alpha}$ is one of the Goldstinos $\theta_{\alpha}^{A}$, let us take it to be $\theta_{\alpha}^{2}$. The coupling induced by the deformation would then be:

$$
\delta S_{\text {inst }}=-\frac{m \pi^{2}}{g_{Y M}} \theta^{2 \alpha} \theta_{\alpha}^{2}
$$

Just as in the gauge theory case, we can bring down this coupling in order to saturate the fermionic integration over the $\theta_{\alpha}^{2}$ modes. This integration will give us a factor of $m$ in front of the resulting contribution to the superpotential (the factors of $\pi$ and $g_{Y M}$ get taken care of once we integrate over the rest of the zero modes properly [41]).

The rest of the calculation, once we have dealt with the massive adjoint, is by now well understood (see for example [33] for a careful derivation for the more involved $U(1)$ instanton). The end result is simply given by:

$$
W_{e f f}(\tau)=m M_{s}^{2} \exp (-2 \pi i \tau)
$$

The $M_{s}$ factors come from the measure of integration of the zero modes [28, 32, 33], and the exponential suppression comes from the volume of the cycle wrapped by the instanton. 


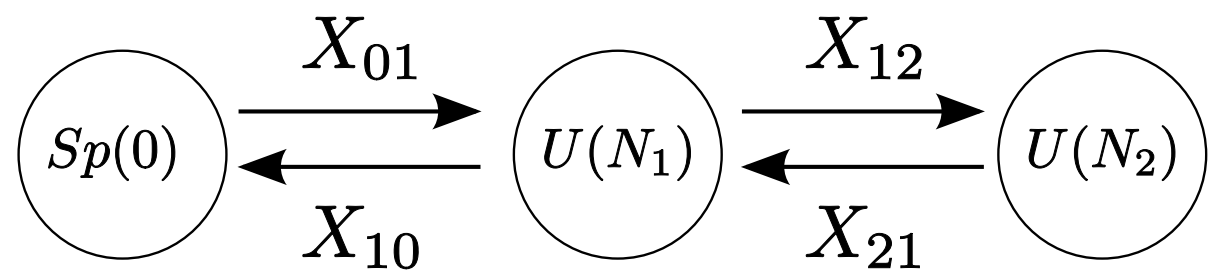

Figure 1: Quiver for the orientifolded orbifold of the conifold we discuss in the main text. We have omitted the part of the quiver to the right of $U\left(N_{2}\right)$, which will not be relevant for our discussion.

In order to match with the matrix model result we need to relate the low energy physical $\Lambda$ appearing in (3.5) with the microscopic scales appearing in (3.9). This goes as follows. The physical scale for the theory before integrating out the adjoint is given in terms of the string scale quantities as:

$$
\Lambda_{a d j}^{2}=M_{S}^{2} e^{-2 \pi i \tau}
$$

The $m$ prefactor gives the matching relation between the theory before and after integrating out the adjoint:

$$
\Lambda^{3}=\Lambda_{a d j}^{2} m
$$

The resulting superpotential can thus be written as:

$$
W=\Lambda^{3}
$$

matching (3.5) beautifully.

\section{A quiver example: $\mathbb{Z}_{n}$ orbifold of the conifold}

Let us proceed to the more interesting case in which the D-brane instanton intersects some space-time filling branes. In this case, as discussed in [7, 8, 9], there are fermionic zero modes arising from strings stretching between the instanton and the brane. Integration over them typically induces interesting couplings in the world volume theory of the brane, which might be potentially relevant for model building. They generically give rise to couplings with are forbidden in perturbation theory.

In this section we will focus on a model which is easy to analyze using matrix model techniques. It is the theory corresponding to branes at the singularity of an abelian orbifold of the conifold. These theories can be easily obtained from $A_{n}$ orbifold quivers by giving large masses to the adjoints. The analysis we do here is easily generalizable to $\mathcal{N}=2$ quiver theories with superpotentials for the adjoints, as in the original paper of Dijkgraaf and Vafa [2]. We will choose an assignment of ranks of the quiver such that the orientifolded node is empty, giving rise to one of the $S p(0)$ factors we have been describing.

The relevant quiver diagram is shown in Figure 1, where we are omitting the part of the quiver that will not be relevant for us. The nodes of the diagram denote gauge factors, and the arrows denote bifundamental chiral multiplets. The theory also has a quartic superpotential, given for generic ranks in the nodes by:

$$
W=\operatorname{Tr} X_{01} X_{12} X_{21} X_{10}
$$


In our case, since we are setting the rank of the $S p$ node to zero, $X_{01}$ and $X_{10}$ vanish, and $W=0$. Nevertheless, as we saw in Section 2.1, the ranks that go into the matrix model computation are unrelated to those in the physical string. This means that we can compute the effective superpotential keeping $X_{01}, X_{10}$ and $W$ in the game, and treating them as if we had $S p(K)$ with $K>0$. The only place where the actual rank of $S p(K)$ enters in the matrix model computation is in determining the prefactor in the Veneziano Yankielowicz term, as in eq. (2.5).

Let us proceed to compute the low energy superpotential from the matrix model point of view. We want to obtain the effect induced by one instanton in the $S p(0)$ node. One way we could go about computing this effect would be by applying the prescription in [2] for $A_{n}$ quivers and taking the limit where the adjoint masses go to infinity, keeping the dynamical scales of the resulting conifold theory finite. See for example 44, 45, 46, 47] for calculations along these lines. Since we are not interested in the full answer, but just the leading term due to $S p(K)$, we can take instead the following shortcut.

The leading term we are interested in corresponds to letting $S p(K)$ confine while taking the rest of the quiver theory to be an spectator. In this way $U\left(N_{1}\right) \times U\left(N_{2}\right)$ can be taken to be a flavor group, and $X_{12}, X_{21}$ are background vevs. From this point of view, we can take (4.1) to mean:

$$
W=\operatorname{Tr} X_{01} X_{12}\left\langle X_{21} X_{12}\right\rangle
$$

In this equation $\left\langle X_{21} X_{12}\right\rangle$ is the mass matrix for $X_{01}, X_{12}$, which we can now integrate out. It is important to take into account the matching relation:

$$
\Lambda_{\text {low }}^{3(K+1)}=\Lambda_{\text {high }}^{3(K+1)-N_{1}} \operatorname{det}\left\langle X_{21} X_{12}\right\rangle
$$

where $\Lambda_{h i g h}$ and $\Lambda_{\text {low }}$ are the dynamical scales of the $S p(K)$ factor before and after integrating out the massive matter.

The remaining theory is $S p(K)$ with no flavors. It confines, and the low energy superpotential is given by:

$$
W=(K+1) \Lambda_{\text {low }}^{3}=(K+1) \Lambda_{\text {high }}^{3-N_{1} /(K+1)} \operatorname{det}\left\langle X_{21} X_{12}\right\rangle^{\frac{1}{K+1}}
$$

We can now use this result computed in the gauge theory with $K>0$, extract the matrix model result for $\partial \mathcal{Z}_{0} / \partial S$, and apply it to the $S p(0)$ case. We obtain the result:

$$
W_{\text {eff }}=\Lambda^{3-N_{1}} \operatorname{det}\left\langle X_{21} X_{12}\right\rangle
$$

Let us now compare this result with the D-brane instanton computation. The technology for dealing with this problem is by now standard [7, 8, 9]. An instanton sitting on the $S p(0)$ node has two neutral zero modes $\theta_{\alpha}$, and two zero modes $\alpha, \beta$ (these are anticommuting scalars living in the fundamental and antifundamental of $U\left(N_{1}\right)$ ) arising from strings going from the instanton to the $U\left(N_{1}\right)$ brane. Essentially the same physics [48 that gives rise to the superpotential (4.1) gives rise to the following coupling in the instanton action:

$$
\delta S_{\text {inst }}=\alpha X_{12} X_{21} \beta
$$


The contribution of the instanton to the low energy effective superpotential is then given by:

$$
W_{e f f}=m M_{s}^{2-N_{1}} e^{-2 \pi i \tau} \int d \alpha d \beta \exp \left(\delta S_{i n s t}\right)
$$

The overall prefactor works exactly as in the conifold case studied in Section 3, and we can identify it with $\Lambda^{3-N_{1}}$ above. The second term can be used to saturate the integrations over $\alpha$ and $\beta$, and gives rise to:

$$
\int d \alpha d \beta \exp \left(\delta S_{\text {inst }}\right)=\operatorname{det} X_{12} X_{21}
$$

reproducing the result (4.5) obtained from the matrix model.

We see that the essential ingredients that went into the previous calculation are those that appeared already in Section 3, where we studied the case of the isolated instanton. Namely, once we considered the glueball superfield for the node containing the instanton dynamical, the matrix model correctly gave us the same answer as the (in general more involved) instanton calculation.

\section{Conclusions and further directions}

In this work we have started the study of D-brane instanton effects from the point of view of matrix models. We found that at least the simplest examples indicate that, in the same way that ordinary gauge non-perturbative effects are captured by a perturbative calculation in the matrix model, more stringy effects are also computed by the matrix model. We checked this result in some detail for isolated conifolds, and for quiver gauge theories admitting a matrix model description.

There are many ways in which one could extend the results in this note, let us list here a few of them.

We have restricted ourselves to matching the matrix model and the D-brane instanton results just up to leading order in the instanton expansion, namely the one (fractional) instanton sector. This simplified the calculations enormously, reducing the problem to writing down the Veneziano-Yankielowicz superpotential for the instanton node and extremizing the complete superpotential. This is already a good sign, and indicates that the matrix model formalism might be significantly simpler to deal with (when it is applicable) than the D-brane instanton calculus, which is sometimes involved even in the one-instanton sector.

In this same line of argument, one technical difficulty to match the higher orders in the matrix model perturbation expansion with non-perturbative effects in string theory is that we will have to deal with multi-(fractional) instanton effects in the string side, and these are somewhat cumbersome to deal with using standard (D-brane) instanton calculus. See however 41] for a review of some powerful techniques for dealing with multi-instantons in the case of gauge theory with extended susy, [49] for progress in the $\mathcal{N}=1$ case, and [36, 11, 50, 51, 37, 52, 53 for some recent progress in more stringy systems. It would 
be interesting to check the proposed correspondence for higher orders in the instanton expansion using some of these techniques.

The geometries on which one can apply directly the ideas of Dijkgraaf and Vafa are rather restricted, and not very suitable for model building ${ }^{8}$. Since the time when the DV results appeared there have been very interesting developments in the study of matrix models and topological string theory. Particularly relevant for our story is [54], where a matrix-model inspired formalism is given for computing perturbative amplitudes of the B-model on a very rich set of geometric backgrounds (in particular, this includes mirrors of toric Calabi-Yau spaces). It would be extremely interesting to see if and how D-brane instanton effects appear in this setup.

We would also like to make some preliminary comments on the study of higher Fterms in the context of matrix models (we have succinctly introduced these higher F-terms towards the end of Section 2.2). Recently it has become clear that these higher F-terms form an integral part of the global picture for D-brane instanton effects across moduli space [11, 37, 40]. They have a fascinating interplay with ordinary superpotential terms in order to give a consistent description of non-perturbative effects across the whole moduli space of the compactification. It would be rather interesting to see if and how these higher F-terms are encoded in the matrix model description.

One seemingly reasonable possibility is that these terms are computed by the nonplanar diagrams in the matrix model. A naive and straightforward reason is that we need to compute terms in the effective superpotential with derivatives, or more than two fermions, and it is well known that in the topological string higher genus diagrams are associated with operators of this kind in the physical superstring. This is obviously too sketchy, so it would be good to have a more direct connection between non-planar diagrams and Beasley-Witten higher F-terms. We know that non-planar diagrams encode the effect of the C-deformation of the gluino anticommutation algebra [55], so if non-planar diagrams are also related to higher F-terms then we would have a nice relation between Beasley-Witten higher F-terms and the C-deformation.

As a last remark, all of our discussion has been in the context of type IIB string theory. By mirror symmetry, one could expect that Chern-Simons theory in the appropriate background might also compute the effect of exotic E2 instantons. It would be rather interesting to see how this works.

\section{Acknowledgments}

I am happy to acknowledge interesting and fruitful discussions with Marta Gomez-Reino, Daniel Krefl, Sergio Montañez and Angel Uranga. I also want to thank Nao Hasegawa for kind support and constant encouragement. This work is supported by the High Energy Physics Research Grant DE-FG05-95ER40893-A020.

\footnotetext{
${ }^{8}$ When we are interested just in the superpotential, we can study D-brane instanton contributions also via the geometric transition, as discussed in the introduction and Section 2.1. There have been some interesting model building applications of these ideas recently, starting with [14].
} 


\section{References}

[1] R. Dijkgraaf and C. Vafa, Matrix models, topological strings, and supersymmetric gauge theories, Nucl. Phys. B644 (2002) 3-20, hep-th/0206255.

[2] R. Dijkgraaf and C. Vafa, On geometry and matrix models, Nucl. Phys. B644 (2002) 21-39, hep-th/0207106.

[3] R. Dijkgraaf and C. Vafa, A perturbative window into non-perturbative physics, hep-th/0208048.

[4] R. Dijkgraaf and C. Vafa, $N=1$ supersymmetry, deconstruction, and bosonic gauge theories, hep-th/0302011.

[5] M. Aganagic, K. Intriligator, C. Vafa, and N. P. Warner, The glueball superpotential, Adv. Theor. Math. Phys. 7 (2004) 1045-1101, hep-th/0304271].

[6] K. Intriligator, P. Kraus, A. V. Ryzhov, M. Shigemori, and C. Vafa, On low rank classical groups in string theory, gauge theory and matrix models, Nucl. Phys. B682 (2004) 45-82, hep-th/0311181.

[7] R. Blumenhagen, M. Cvetic, and T. Weigand, Spacetime instanton corrections in $4 D$ string vacua - the seesaw mechanism for D-brane models, Nucl. Phys. B771 (2007) 113-142, hep-th/0609191.

[8] L. E. Ibanez and A. M. Uranga, Neutrino Majorana masses from string theory instanton effects, JHEP 03 (2007) 052, hep-th/0609213.

[9] B. Florea, S. Kachru, J. McGreevy, and N. Saulina, Stringy instantons and quiver gauge theories, JHEP 05 (2007) 024, hep-th/0610003.

[10] O. Aharony and S. Kachru, Stringy Instantons and Cascading Quivers, JHEP 09 (2007) 060, arXiv:0707.3126.

[11] I. Garcia-Etxebarria and A. M. Uranga, Non-perturbative superpotentials across lines of marginal stability, JHEP 01 (2008) 033, arXiv:0711.1430.

[12] A. Amariti, L. Girardello, and A. Mariotti, Stringy Instantons as Strong Dynamics, arXiv:0809.3432.

[13] D. Krefl, A gauge theory analog of some 'stringy' D-instantons, arXiv:0803.2829.

[14] M. Aganagic, C. Beem, and S. Kachru, Geometric Transitions and Dynamical SUSY Breaking, Nucl. Phys. B796 (2008) 1-24, arXiv:0709.4277.

[15] F. Cachazo, M. R. Douglas, N. Seiberg, and E. Witten, Chiral rings and anomalies in supersymmetric gauge theory, JHEP 12 (2002) 071, hep-th/0211170.

[16] R. Dijkgraaf, M. T. Grisaru, C. S. Lam, C. Vafa, and D. Zanon, Perturbative computation of glueball superpotentials, Phys. Lett. B573 (2003) 138-146, hep-th/0211017.

[17] F. Ferrari, On exact superpotentials in confining vacua, Nucl. Phys. B648 (2003) 161-173, hep-th/0210135.

[18] G. Veneziano and S. Yankielowicz, An Effective Lagrangian for the Pure N=1 Supersymmetric Yang-Mills Theory, Phys. Lett. B113 (1982) 231.

[19] S. Kachru, S. H. Katz, A. E. Lawrence, and J. McGreevy, Open string instantons and superpotentials, Phys. Rev. D62 (2000) 026001, hep-th/9912151. 
[20] P. Candelas and X. C. de la Ossa, Comments on Conifolds, Nucl. Phys. B342 (1990) 246-268.

[21] M. Marino, R. Schiappa, and M. Weiss, Nonperturbative Effects and the Large-Order Behavior of Matrix Models and Topological Strings, arXiv:0711.1954.

[22] E. Witten, Chern-Simons gauge theory as a string theory, Prog. Math. 133 (1995) 637-678, hep-th/9207094.

[23] M. Bershadsky, S. Cecotti, H. Ooguri, and C. Vafa, Kodaira-Spencer theory of gravity and exact results for quantum string amplitudes, Commun. Math. Phys. 165 (1994) 311-428, hep-th/9309140.

[24] C. Vafa, Superstrings and topological strings at large N, J. Math. Phys. 42 (2001) 2798-2817, hep-th/0008142.

[25] F. Cachazo, K. A. Intriligator, and C. Vafa, A large $N$ duality via a geometric transition, Nucl. Phys. B603 (2001) 3-41, hep-th/0103067.

[26] H. Ooguri and C. Vafa, Worldsheet derivation of a large $N$ duality, Nucl. Phys. B641 (2002) $3-34$, hep-th/0205297.

[27] L. E. Ibanez, A. N. Schellekens, and A. M. Uranga, Instanton Induced Neutrino Majorana Masses in CFT Orientifolds with MSSM-like spectra, JHEP 06 (2007) 011, arXiv:0704.1079.

[28] M. Billo et. al., Classical gauge instantons from open strings, JHEP 02 (2003) 045, hep-th/0211250.

[29] N. Akerblom, R. Blumenhagen, D. Lust, E. Plauschinn, and M. Schmidt-Sommerfeld, Non-perturbative SQCD Superpotentials from String Instantons, JHEP 04 (2007) 076, hep-th/0612132.

[30] M. Bianchi and E. Kiritsis, Non-perturbative and Flux superpotentials for Type I strings on the $Z_{3}$ orbifold, Nucl. Phys. B782 (2007) 26-50, hep-th/0702015.

[31] R. Argurio, M. Bertolini, G. Ferretti, A. Lerda, and C. Petersson, Stringy Instantons at Orbifold Singularities, JHEP 06 (2007) 067, arXiv:0704.0262.

[32] M. Bianchi, F. Fucito, and J. F. Morales, D-brane Instantons on the $T^{6} / Z_{3}$ orientifold, JHEP 07 (2007) 038, arXiv:0704.0784.

[33] C. Petersson, Superpotentials From Stringy Instantons Without Orientifolds, JHEP 05 (2008) 078, arXiv:0711.1837.

[34] C. Beasley and E. Witten, New instanton effects in supersymmetric QCD, JHEP 01 (2005) 056, hep-th/0409149.

[35] C. Beasley and E. Witten, New instanton effects in string theory, JHEP 02 (2006) 060, hep-th/0512039.

[36] R. Blumenhagen, M. Cvetic, R. Richter, and T. Weigand, Lifting D-Instanton Zero Modes by Recombination and Background Fluxes, JHEP 10 (2007) 098, arXiv:0708.0403.

[37] I. Garcia-Etxebarria, F. Marchesano, and A. M. Uranga, Non-perturbative F-terms across lines of BPS stability, JHEP 07 (2008) 028, arXiv:0805.0713.

[38] M. Billo' et. al., Flux interactions on D-branes and instantons, arXiv:0807.1666. 
[39] M. Billo' et. al., Non-perturbative effective interactions from fluxes, arXiv:0807.4098.

[40] A. M. Uranga, D-brane instantons and the effective field theory of flux compactifications, arXiv:0808.2918.

[41] N. Dorey, T. J. Hollowood, V. V. Khoze, and M. P. Mattis, The calculus of many instantons, Phys. Rept. 371 (2002) 231-459, hep-th/0206063].

[42] E. Witten, Small Instantons in String Theory, Nucl. Phys. B460 (1996) 541-559, hep-th/9511030.

[43] M. R. Douglas, Gauge Fields and D-branes, J. Geom. Phys. 28 (1998) 255-262, hep-th/9604198.

[44] S. Seki, Comments on quiver gauge theories and matrix models, Nucl. Phys. B661 (2003) 257-272, hep-th/0212079.

[45] R. Casero and E. Trincherini, Phases and geometry of the $N=1$ A(2) quiver gauge theory and matrix models, JHEP 09 (2003) 063, hep-th/0307054.

[46] S. Chiantese, A. Klemm, and I. Runkel, Higher order loop equations for $A(r)$ and $D(r)$ quiver matrix models, JHEP 03 (2004) 033, hep-th/0311258].

[47] Y. Ookouchi, $N=1$ gauge theory with flavor from fluxes, JHEP 01 (2004) 014, hep-th/0211287.

[48] S. Kachru and D. Simic, Stringy Instantons in IIB Brane Systems, arXiv:0803.2514.

[49] F. Fucito, J. F. Morales, R. Poghossian, and A. Tanzini, $N=1$ superpotentials from multi-instanton calculus, JHEP 01 (2006) 031, hep-th/0510173].

[50] R. Blumenhagen and M. Schmidt-Sommerfeld, Power Towers of String Instantons for $N=1$ Vacua, JHEP 07 (2008) 027, arXiv:0803.1562.

[51] M. Cvetic, R. Richter, and T. Weigand, (Non-)BPS bound states and D-brane instantons, JHEP 07 (2008) 012, arXiv:0803.2513.

[52] P. G. Camara and E. Dudas, Multi-instanton and string loop corrections in toroidal orbifold models, JHEP 08 (2008) 069, arXiv:0806.3102.

[53] D. Gaiotto, G. W. Moore, and A. Neitzke, Four-dimensional wall-crossing via three-dimensional field theory, arXiv:0807.4723.

[54] V. Bouchard, A. Klemm, M. Marino, and S. Pasquetti, Remodeling the B-model, arXiv:0709.1453.

[55] H. Ooguri and C. Vafa, The C-deformation of gluino and non-planar diagrams, Adv. Theor. Math. Phys. 7 (2003) 53-85, hep-th/0302109. 